\title{
Benign External Ear Neoplasm
}

National Cancer Institute

\section{Source}

National Cancer Institute. Benign External Ear Neoplasm. NCI Thesaurus. Code C6807.

A non-metastasizing neoplasm that arises from the external ear. Representative examples include ceruminous adenoma, polyp, and lipoma. 